\title{
Establishment of reference intervals for serum
}

\section{[-2]proPSA (p2PSA), \%p2PSA and prostate health index in healthy men}

This article was published in the following Dove Press journal: OncoTargets and Therapy

\author{
Zhi-Yu Wu' \\ Cheng Yang' \\ Jie Luo' \\ Shao-Li Deng' \\ Bin Wu' \\ Ming Chen ${ }^{1-3}$ \\ 'Department of Clinical Laboratory \\ Medicine, Daping Hospital, Army Medical \\ University, Chongqing 400042, People's \\ Republic of China; ${ }^{2}$ College of Pharmacy \\ and Laboratory Medicine, Army Medical \\ University, Chongqing 400038, People's \\ Republic of China; ${ }^{3}$ State Key Laboratory \\ of Trauma, Burn and Combined Injury, \\ Army Medical University, Chongqing \\ 400042, People's Republic of China
}

Correspondence: Ming Chen Department of Clinical Laboratory Medicine, Daping Hospital, Army Medical University, No. 10, Changjiang Branch Road, Yuzhong District, Chongqing 400042, People's Republic of China

Tel +86 I3। $1023 \quad$ I375

Fax +862368757601

Email chming197I@I26.com
Background: Prostate cancer (PCa) is the second most common cancer among men worldwide. Recent research has identified [-2]proPSA (p2PSA), \%p2PSA and prostate health index (phi) as new biomarkers for the early diagnosis and grading of PCa. However, few studies have used these parameters in a healthy population. In this study, we aimed to establish reference intervals (RIs) for p2PSA, \%p2PSA and phi in healthy men based on age stratification.

Methods: Between April 2016 and March 2018, healthy subjects were recruited. Healthy men were then stratified into four age groups: $<40$ years, 40-49 years, 50-59 years and $\geq 60$ years. Total PSA (tPSA), free PSA (fPSA), \%fPSA, p2PSA, \%p2PSA and phi were measured and RIs were established for p2PSA, \%p2PSA and phi.

Results: In total, 732 healthy men were used for analysis. The RIs of phi were 9.77-48.44 for $<40$ years of age, 9.85-65.28 for 40-49 years of age, 9.98-39.72 for 50-59 years of age and $8.16-40.76$ for $\geq 60$ years of age. The reference values at the age of $40-49$ years were generally higher than those at $\geq 60$ years of age.

Conclusions: Age-specific RIs for p2PSA, \%p2PSA and phi were established in this study. This first set of established RIs will be invaluable for physicians to make precise medical decisions and carry out appropriate medical interventions.

Keywords: PSA, p2PSA, prostate health index, phi, prostate cancer, reference interval

\section{Introduction}

Prostate cancer ( $\mathrm{PCa}$ ) is one of the most common malignancies in males worldwide and one of the principal causes of death from cancer in the male population. ${ }^{1}$ The current diagnosis of PCa depends on transrectal ultrasonography (TRUS) and biopsy. However, both of these techniques are invasive and are not easy to carry out, and are therefore not conducive to an early diagnosis. The most common practice is to perform routine PSA (prostate specific antigen) testing; PSA is a valid tumor marker for the swift and efficient screening of PCa. This test has increased the detection rates of early-stage $\mathrm{PCa}$ and reduced the mortality rates associated with this condition. ${ }^{2-4}$ However, PCa screening has been associated with unnecessary biopsies, over-diagnosis, and subsequent over-treatment. ${ }^{5,6}$ Due to the low specificity of the PSA test, the current guidelines do not recommend PSAbased PCa screening and as a consequence, researchers are actively searching for more specific tumor biomarkers. In recent years, [-2]proPSA (p2PSA), \%p2PSA and prostate health index (phi) have gradually gained acceptance by the scientific 
community and have become the primary biomarkers investigated for the diagnosis of PCa. Research studies have already been carried out on p2PSA, \%p2PSA and phi and the majority of these studies were performed in PCa patients with total prostate-specific antigen (tPSA) levels of $4-10 \mathrm{ng} / \mathrm{mL}$ (referred to as the "gray zone") and in patients with PSA levels $>10 \mathrm{ng} / \mathrm{mL}^{7-11}$ While tPSA may have early diagnostic and screening value, there is very little evidence in this regard. Research populations selected based on abnormal tPSA may be biased. Consequently, it is necessary to detect additional biomarkers such as p2PSA, \%p2PSA and phi in healthy population. Consequently, the establishment of RIs is of great significance and clinical application value for the early diagnosis of PCa. Unfortunately, there are few research reports describing this style of protocol, and there is limited information relating to the reference limits for this group of men. ${ }^{12-14}$ Consequently, the purpose of this research study was to establish RIs for p2PSA, \%p2PSA and $p h i$ in healthy men, stratified by age.

\section{Materials and methods Subjects}

This study complied with the Code of Ethics of the World Medical Association, was approved by the Hospital Ethics Committee (Reference Number: 201433) and was carried out between April 2016 and March 2018. After obtaining informed consent, subjects were recruited following physical examination at Daping Hospital in Chongqing, China. Inclusion criteria were as follows: (1) a tPSA concentration $<4 \mathrm{ng} / \mathrm{mL}$ and (2) a negative result from transabdominal prostate ultrasound. Negative ultrasound results were defined as a normal prostate size, without any positive manifestations, including prostate nodules, bilateral asymmetry of the outer glands, increased blood flow signals in the glands, unclear boundaries between the inner and outer glands and abnormal morphology. Exclusion criteria were: (1) a prior history of $\mathrm{PCa}$; (2) acute or chronic bacterial prostatitis; (3) active urinary tract infections; (4) previous transurethral resection of the prostate; and (5) the use of drugs or other therapies within 6 weeks preceding blood collection that might have influenced PSA concentrations. The subjects recruited were stratified into four age groups: $<40$ years, $40-49$ years, $50-59$ years and $\geq 60$ years.

\section{Sample collection and analysis}

Blood samples were collected before any prostatic manipulations that could have caused a transient increase in biomarker levels. Following blood collection, serum was immediately separated by centrifugation and stored at $-70{ }^{\circ} \mathrm{C}$ to await analysis. Maximum storage time was approximately 4 months. Prior to analysis, the stored serum samples were thawed at room temperature, mixed and analyzed using a Beckman Coulter Unicel DXI 800 automatic immunity analyzer (Beckman Coulter, Brea, CA, USA), along with the Hybritech-calibrated Access tPSA, fPSA and p2PSA assays. In total, we assayed the levels of six biomarkers in each serum sample, which included tPSA, fPSA, \%fPSA, p2PSA, \%p2PSA and phi.

\section{Data collection and statistics}

p2PSA derivatives were calculated as shown in equations (1) and (2) below.

$$
\begin{gathered}
\% \mathrm{p} 2 \mathrm{PSA}=\mathrm{p} 2 \mathrm{PSA} * 100 /(\mathrm{fPSA} * 1000) \\
\text { phi }=(\mathrm{p} 2 \mathrm{PSA} / \mathrm{fPSA}) * \mathrm{tPSA}^{0.5}
\end{gathered}
$$

Demographic information and results were recorded electronically using Statistical Product and Service Solution (SPSS) Version 22.0 for Windows (SPSS, Chicago, IL, USA) and presented with GraphPad Prism Version 7 for Windows (GraphPad Software, San Diego, CA, USA). Prior to calculating RIs, the outliers were identified by the Dixon's test and interpreted by Reed's criteria in which the absolute difference between an extreme observation and the next largest observation (D) was divided by the range of all observations (R). ${ }^{15}$ If the difference $\mathrm{D}$ was equal to or greater than one-third of the range of $\mathrm{R}$, the extreme value was deleted. ${ }^{16}$ To ascertain whether separate age-specific reference intervals should be used in clinical practice, we determined $90 \%$ confidence intervals for each biomarker's upper and lower reference limits. Standard descriptive statistics were used. Non-normally distributed variables are presented as medians and interquartile ranges (IQR), while normally distributed data were presented as means and standard deviations. When data were not normally distributed, comparisons were made using the Mann-Whitney $U$ test for comparison between two groups or the Kruskal-Wallis test with Dunn's multiple comparisons for multiple groups. The level of significance was set at a $p$-value $<0.05$. Reference values were calculated by non-parametric 
methods following guidelines (C28-A3c) published by the Clinical and Laboratory Standards Institute (CLSI). ${ }^{17}$

\section{Results}

\section{Patient recruitment}

In total, 1707 subjects with tPSA levels $<4 \mathrm{ng} / \mathrm{mL}$ were recruited for this study. Of these, 190 cases were missing ultrasound results. Consequently, 1517 of the recruited cases had the necessary data for inclusion. However, only 732 cases were ultrasound-negative with tPSA levels $<4 \mathrm{ng} / \mathrm{mL}$ and could be included in this study. These patients accounted for 48.25\% (732/ 1517) of the recruited cases (Table 1). The proportions of males with negative ultrasound results in the $<40$ years, 40-49 years, 50-59 years and $\geq 60$ years age groups were 86.54\% (135/156), 70.83\% (306/ 432), 46.63\% (166/356) and 21.82\% (125/573), respectively. Quantitative data and the proportional distribution of the recruited patients in the four age groups are shown in Figure 1. Notably, the proportion of patients who had positive ultrasound results increased with age $(p<0.0001)$.

\section{Changes in the serum levels of six biomarkers in healthy males}

According to Dixon and Reed's criteria, ${ }^{15,16}$ six outliers were detected, which included one case in the $<40$ years age group, one case in the 40-49 years age group, two cases in the 5059 years age group, and two cases in the $\geq 60$ years age group. Consequently, 726 cases were finally used to establish the RIs.

The means, standard errors of the mean, and selected percentiles for tPSA, fPSA, \%fPSA, p2PSA, \%p2PSA and phi are shown, stratified by age group, in Table 2. Boxplot and curves, depicting age-related distribution, are shown in Figures 2 and 3. In general, fPSA, \%fPSA and p2PSA curves for healthy males showed similar trends; decreasing with age $(p<0.05)$ and rising slightly at the age of $\geq 60$ years. Similarly, the curves of tPSA, \%p2PSA and phi showed the same performance as each other with the highest peaks appearing during 40-49 years of age. There were no significant differences in tPSA or $\%$ p2PSA across the different age groups $(p>0.05)$. In addition, there was no significant difference between the two groups in terms of phi, although significant differences existed between the different age groups $(p=0.0491)$.

Table I Physical examination findings in the population of recruited patients

\begin{tabular}{|l|l|l|l|l|l|l|}
\hline \multicolumn{2}{|l|}{ Examination Findings } & \multicolumn{4}{l|}{ Age groups (years) } & Total \\
\cline { 3 - 7 } & $\mathbf{4 4 0}$ & $\mathbf{4 0 - 4 9}$ & $\mathbf{5 0 - 5 9}$ & $\geq \mathbf{6 0}$ & \\
\hline tPSA <4 ng/mL & $\mathrm{n}(\%)$ & $156(10.28)$ & $432(28.48)$ & $356(23.47)$ & $573(37.77)$ & $1517(100)$ \\
Ultrasound results (-) & $\mathrm{n}(\%)$ & $135(86.54)$ & $306(70.83)$ & $166(46.63)$ & $125(21.82)$ & $732(48.25)$ \\
Ultrasound results (+) & $\mathrm{n}(\%)$ & $21(13.46)$ & $126(29.17)$ & $190(53.37)$ & $\mathbf{4 4 8}(78.18)$ & $785(51.75)$ \\
\hline
\end{tabular}

Notes: Ultrasound results (-) defined as normal prostate size, without any positive manifestations including prostate nodules, bilateral asymmetry of the outer glands, increased blood flow signals in the glands, unclear boundaries between the inner and outer glands and abnormal morphology.

Abbreviation: tPSA, total prostate-specific antigen.

A

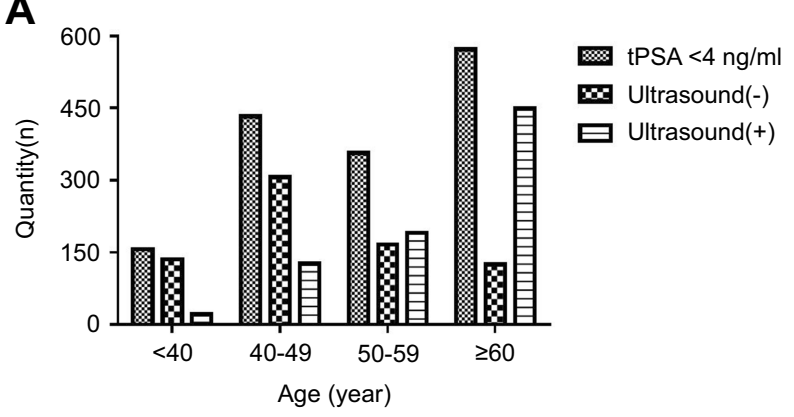

B

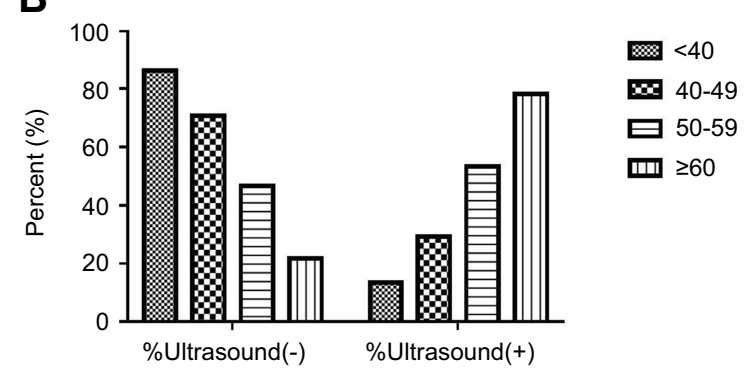

Figure I (A) Quantitative distribution of subjects in four different age groups. (B) Proportion of Ultrasound results (-) and Ultrasound results (+) subjects in four different age groups. Notes: Ultrasound $(-)$ defined as normal prostate size, without any positive manifestations including prostate nodules, bilateral asymmetry of the outer glands, increased blood flow signals in the glands, unclear boundaries between the inner and outer glands, abnormal morphology; Ultrasound (+) defined as oversize prostate, or with manifestations including inflammatory change, prostate nodules, bilateral asymmetry of the outer glands, increased blood flow signals in the glands, unclear boundaries between the inner and outer glands, abnormal morphology.

Abbreviation: tPSA, total prostate-specific antigen. 
Table 2 Mean, standard deviation (SD) of the mean, and selected percentiles for PSA tests in healthy men of various ages

\begin{tabular}{|c|c|c|c|c|c|c|c|c|c|c|}
\hline & \multirow[t]{2}{*}{ Age group (year) } & \multirow[t]{2}{*}{$\mathbf{n}$} & \multirow[t]{2}{*}{ Mean (SD) } & \multicolumn{7}{|c|}{ Percentiles } \\
\hline & & & & 5th & 10th & 25th & 50th & 75th & 90th & 95th \\
\hline \multirow[t]{6}{*}{ tPSA (ng/mL) } & All ages & 726 & $0.92(0.60)$ & 0.31 & 0.38 & 0.52 & 0.79 & 1.10 & 1.61 & 2.15 \\
\hline & $<40$ & 134 & $0.93(0.62)$ & 0.32 & 0.41 & 0.55 & 0.76 & 1.14 & 1.79 & 2.20 \\
\hline & $40-49$ & 305 & $0.92(0.55)$ & 0.33 & 0.41 & 0.56 & 0.80 & 1.10 & 1.53 & 2.02 \\
\hline & $50-59$ & 164 & $0.90(0.57)$ & 0.32 & 0.39 & 0.51 & 0.77 & 1.13 & 1.62 & 2.12 \\
\hline & $\geq 60$ & 123 & $0.94(0.72)$ & 0.20 & 0.27 & 0.44 & 0.78 & 1.10 & 1.83 & 2.88 \\
\hline & $p$-value & \multicolumn{9}{|c|}{0.5856 (NS) } \\
\hline \multirow[t]{6}{*}{ fPSA (ng/mL) } & All ages & 726 & $0.31(0.17)$ & 0.11 & 0.14 & 0.20 & 0.28 & 0.40 & 0.53 & 0.62 \\
\hline & $<40$ & 134 & $0.34(0.15)$ & 0.15 & 0.19 & 0.23 & 0.32 & 0.43 & 0.55 & 0.62 \\
\hline & $40-49$ & 305 & $0.32(0.17)$ & 0.12 & 0.15 & 0.20 & 0.28 & 0.39 & 0.52 & 0.62 \\
\hline & $50-59$ & 164 & $0.29(0.17)$ & 0.10 & 0.13 & 0.18 & 0.24 & 0.37 & 0.54 & 0.65 \\
\hline & $\geq 60$ & 123 & $0.30(0.18)$ & 0.08 & 0.10 & 0.16 & 0.27 & 0.40 & 0.53 & 0.62 \\
\hline & $p$-value & \multicolumn{9}{|c|}{$0.0009(* * *)$} \\
\hline \multirow[t]{6}{*}{ \%fPSA } & All ages & 726 & $0.38(0.14)$ & 0.17 & 0.21 & 0.28 & 0.36 & 0.47 & 0.56 & 0.64 \\
\hline & $<40$ & 134 & $0.43(0.15)$ & 0.18 & 0.22 & 0.31 & 0.43 & 0.53 & 0.64 & 0.70 \\
\hline & $40-49$ & 305 & $0.38(0.13)$ & 0.18 & 0.22 & 0.28 & 0.35 & 0.46 & 0.55 & 0.63 \\
\hline & $50-59$ & 164 & $0.35(0.13)$ & 0.16 & 0.20 & 0.26 & 0.33 & 0.45 & 0.53 & 0.58 \\
\hline & $\geq 60$ & 123 & $0.36(0.13)$ & 0.15 & 0.20 & 0.27 & 0.34 & 0.44 & 0.52 & 0.60 \\
\hline & $p$-value & \multicolumn{9}{|c|}{$<0.0001(* * *)$} \\
\hline \multirow[t]{6}{*}{ p2PSA (pg/mL) } & All ages & 726 & $6.75(4.20)$ & 2.20 & 2.87 & 4.01 & 5.80 & 8.37 & 11.52 & 14.30 \\
\hline & $<40$ & 134 & 7.38 (4.59) & 2.73 & 3.31 & 4.87 & 6.54 & 8.92 & 11.56 & 15.75 \\
\hline & $40-49$ & 305 & 7.21 (4.54) & 2.40 & 3.18 & 4.10 & 6.12 & 9.07 & 12.17 & 16.65 \\
\hline & $50-59$ & 164 & $6.04(3.57)$ & 2.24 & 2.49 & 3.71 & 5.21 & 7.26 & 11.25 & 14.11 \\
\hline & $\geq 60$ & 123 & $5.84(3.28)$ & 1.79 & 2.16 & 3.39 & 5.33 & 7.70 & 10.29 & 12.79 \\
\hline & $p$-value & \multicolumn{9}{|c|}{$0.0001(* * *)$} \\
\hline \multirow[t]{6}{*}{$\%$ p2PSA } & All ages & 726 & $2.25(1.07)$ & 1.36 & 1.49 & 1.72 & 2.07 & 2.45 & 3.02 & 3.60 \\
\hline & $<40$ & 134 & $2.17(0.88)$ & 1.31 & 1.50 & 1.71 & 1.97 & 2.39 & 2.67 & 3.71 \\
\hline & $40-49$ & 305 & $2.37(1.32)$ & 1.42 & 1.56 & 1.81 & 2.10 & 2.51 & 3.21 & 3.92 \\
\hline & $50-59$ & 164 & $2.17(0.85)$ & $\mathrm{I} .40$ & 1.47 & 1.67 & 2.03 & 2.44 & 2.94 & 3.20 \\
\hline & $\geq 60$ & 123 & $2.15(0.74)$ & 1.24 & 1.36 & 1.58 & 2.09 & 2.53 & 3.27 & 3.44 \\
\hline & $p$-value & \multicolumn{9}{|c|}{0.0730 (NS) } \\
\hline \multirow[t]{6}{*}{ phi } & All ages & 726 & $20.28(10.4 I)$ & 10.37 & 11.63 & 14.52 & 18.46 & 23.07 & 28.82 & 35.86 \\
\hline & $<40$ & 134 & $19.95(9.66)$ & 10.37 & 11.00 & 13.72 & 17.77 & 22.36 & 30.90 & 36.56 \\
\hline & $40-49$ & 305 & $21.68(12.86)$ & 11.19 & 12.59 & 15.17 & $19.5 \mid$ & 23.69 & 30.45 & 39.83 \\
\hline & $50-59$ & 164 & $19.15(7.05)$ & 10.70 & 11.70 & 14.34 & 17.20 & 22.99 & 27.75 & 31.08 \\
\hline & $\geq 60$ & 123 & $18.66(7.35)$ & 8.66 & 10.19 & 13.65 & 16.95 & 22.83 & 26.80 & 32.55 \\
\hline & $p$-value & \multicolumn{9}{|c|}{$0.0491\left(^{*}\right)$} \\
\hline
\end{tabular}

Notes: NS indicates no significance; *indicates $p<0.05$; ***indicates $p<0.001$.

Abbreviations: tPSA, total prostate-specific antigen; fPSA, free prostate-specific antigen; p2PSA, [-2]proPSA; phi, prostate health index; NS, not significant.

Age-based reference intervals of p2PSA, $\%$ p2PSA and phi in healthy males

RIs (2.5th to 97.5th percentiles) for p2PSA, \%p2PSA and phi in the overall population were $1.88-17.40 \mathrm{pg} / \mathrm{mL}$ and $1.26-4.54,9.48-45.50$, respectively. RIs for the four different age groups are summarized in Table 3. Several studies have indicated that these markers, including tPSA and p2PSA, would change with age, ${ }^{12,13,18,19}$ the present study provides further supporting evidence for this. Most of the $90 \%$ confidence intervals corresponding to the reference 
A
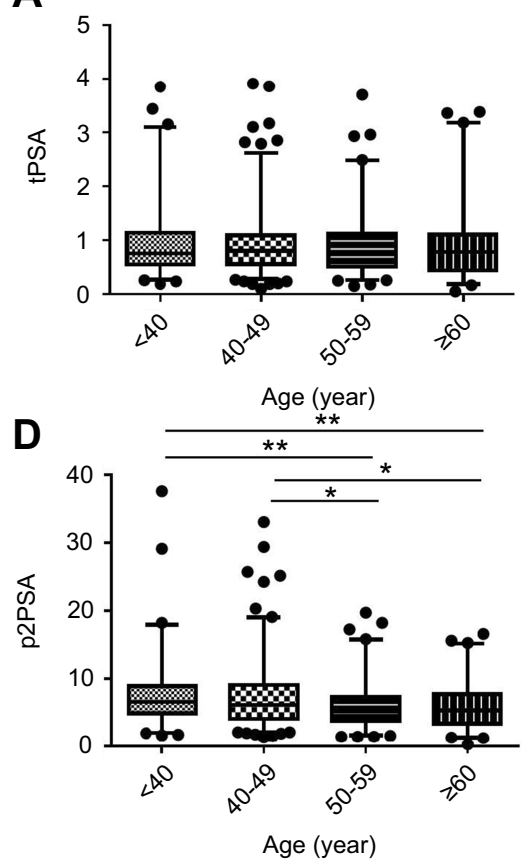

B

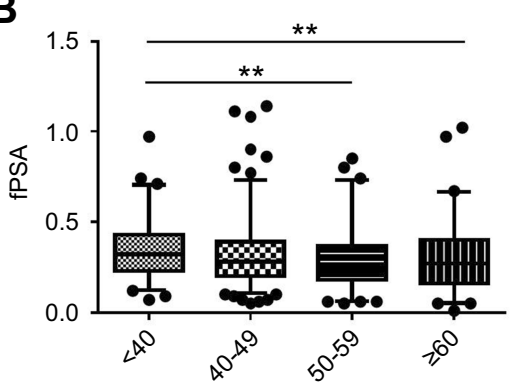

Age (year)

E

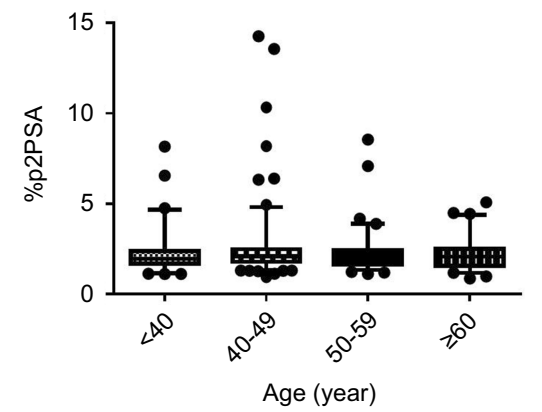

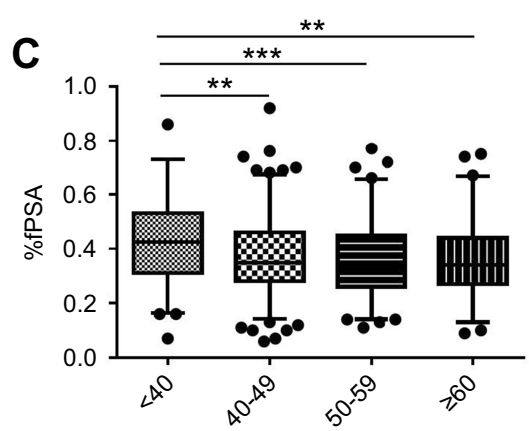

Age (year)

$\mathbf{F}$

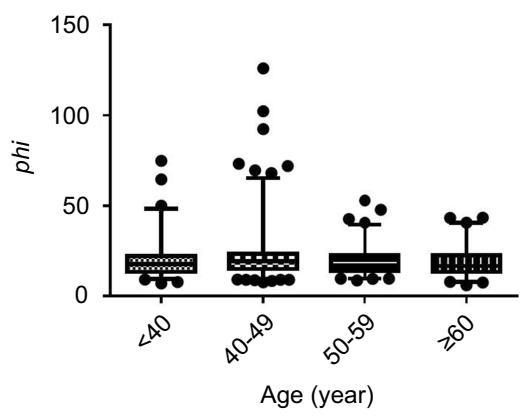

Figure 2 Serum levels of (A) tPSA, (B) fPSA, (C) \%fPSA, (D) p2PSA, (E) \%p2PSA and (F) phi in healthy males among the four different age groups. Boxes and horizontal lines indicate interquartile ranges and medians, respectively; whiskers stretch from the 2.5th to 97.5th percentiles for each age group. Notes: *indicates $p<0.05$; **indicates $p<0.01$; ***indicates $p<0.001$.

Abbreviations: tPSA, total prostate-specific antigen; fPSA, free prostate-specific antigen; p2PSA, [-2]proPSA; phi, prostate health index.

A

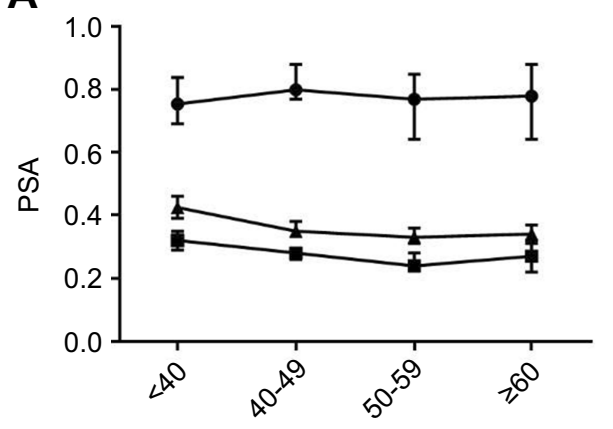

Age (year)
B
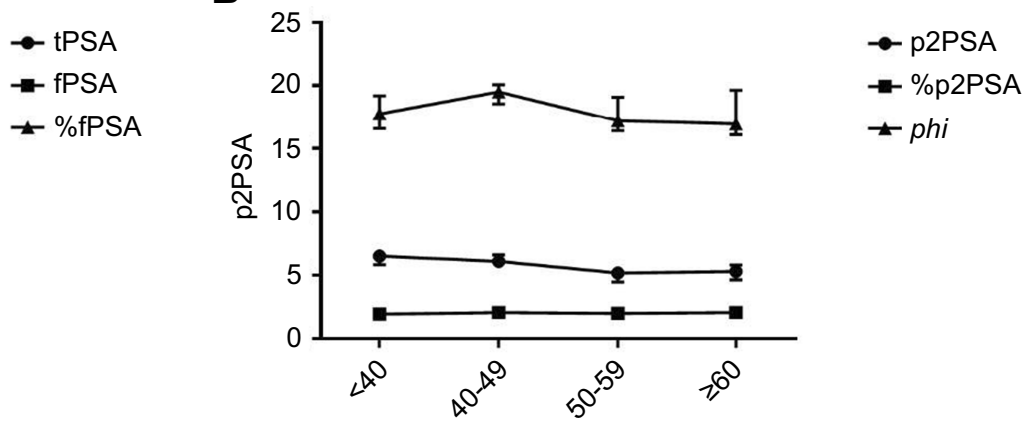

Age (year)

Figure 3 The age-related distribution curves of PSA (A), p2PSA and phi (B). Dots, squares and triangles indicate medians, respectively; whiskers stretch from the -95\% confidence interval $(\mathrm{Cl})$ to the $95 \% \mathrm{Cl}$ for each age group.

Abbreviations: tPSA, total prostate-specific antigen; fPSA, free prostate-specific antigen; p2PSA, [-2]proPSA; phi, prostate health index.

limits overlapped with one another, indicating that the RIs were applicable to the three biomarkers were, in general, not different among the entire population or the four age groups. There were eight non-overlapping $90 \%$ confidence intervals found in the $40-49$ years cohort and the $\geq 60$ years cohort, including the upper reference limit of p2PSA, the lower reference limit of \%p2PSA, and both the upper and lower reference limits of $p h i$ for both of these age groups.
The data showed that the reference limits at 40-49 years of age were generally higher than those at $\geq 60$ years of age.

\section{Discussion}

RIs are critical for clinical laboratory test interpretation and patient care. The test results themselves are of little value unless the report has an appropriate RI or medical decision limit. To the best of our knowledge, this 
Table 3 Reference intervals for p2PSA, \% p2PSA and phi

\begin{tabular}{|c|c|c|c|c|}
\hline \multirow[t]{2}{*}{ Biomarker } & \multirow[t]{2}{*}{ Age group (years) } & \multirow[t]{2}{*}{$\mathbf{n}$} & \multicolumn{2}{|l|}{ Percentile } \\
\hline & & & 2.5th & 97.5th \\
\hline \multirow[t]{5}{*}{ p2PSA (pg/mL) } & All ages & 726 & I.88 (I.72-2.07) & $17.40(|5.83-| 8.8 \mid)$ \\
\hline & $<40$ & 134 & $2.02(1.60-2.89)$ & 17.93 (15.17-37.58) \\
\hline & $40-49$ & 305 & $2.08(1.74-2.34)$ & $19.02(17.22-25.70)^{*}$ \\
\hline & $50-59$ & 164 & $1.60(1.41-2.25)$ & 15.75 (|4.00-19.7I) \\
\hline & $\geq 60$ & 123 & $\mathrm{I} .28(0.28-\mathrm{I} .8 \mathrm{I})$ & $15.15(11.85-16.57)^{*}$ \\
\hline \multirow[t]{5}{*}{$\%$ P2PSA } & All ages & 726 & $1.26(1.21-1.33)$ & $4.54(3.94-4.77)$ \\
\hline & $<40$ & 134 & $1.17(1.14-1.34)$ & $4.69(3.52-8.17)$ \\
\hline & $40-49$ & 305 & $1.34(1.29-1.39)^{*}$ & $4.82(4.12-10.33)$ \\
\hline & $50-59$ & 164 & $1.35(I .15-1.41)$ & 3.91 (3.18-8.56) \\
\hline & $\geq 60$ & 123 & $1.21(0.89-1.26)^{*}$ & $4.4 I(3.4 I-5.09)$ \\
\hline \multirow[t]{5}{*}{ phi } & All ages & 726 & $9.48(8.92-10.09)$ & $45.50(40.0 \mathrm{I}-52.84)$ \\
\hline & $<40$ & 134 & 9.77 (7.25-10.44) & $48.44(34.96-74.88)$ \\
\hline & $40-49$ & 305 & $9.85(9.00-10.98)^{*}$ & $65.28(45.50-92.38)^{*}$ \\
\hline & $50-59$ & 164 & $9.98(8.89-10.86)$ & $39.72(30.9 I-53.09)$ \\
\hline & $\geq 60$ & 123 & $8.16(6.26-8.92)^{*}$ & $40.76(30.40-43.52)^{*}$ \\
\hline
\end{tabular}

Notes: Values in brackets indicate the $90 \%$ confidence interval $(\mathrm{Cl})$ of the corresponding reference limits. *Indicates non-overlapping $90 \%$ Cls between the two groups. Abbreviations: p2PSA, [-2]proPSA; phi, prostate health index.

represents the first study to establish reference intervals for p2PSA, \%p2PSA and phi in a healthy male population.

Previous studies from across the world have focused on males over 40 years of age, particularly those of older age, and have only concerned the specific characteristics of prostate disease. ${ }^{20,21}$ A major shortcoming of these previous studies is that biomarkers of prostate disease were not characterized in the healthy male population. This made it impossible to set accurate and specific ranges for biomarkers of prostate disease in normal healthy males. The aim of the present study was to investigate the dynamic trends of p2PSA, \%p2PSA and phi across a healthy population of males with a wide range of ages, featuring both young and elderly patients. Our primary aim was to establish RIs for p2PSA, \%p2PSA and phi within the normal healthy male population. Therefore, in addition to investigating subjects with normal levels of tPSA $(<4 \mathrm{ng} / \mathrm{mL})$, our selection of healthy men also meant that we were investigating subjects who were ultrasound negative. This is why our results and conclusions differ from previous reports using healthy populations. ${ }^{12,13}$ Subjects included in previous studies were recruited on the basis of their normal IPSA, yet we found that the proportion of subjects with a normal prostate size (ie, no prostatic hyperplasia or hypertrophy) was only $48.25 \%$ in this study. When stratified by age, the proportions of males with an enlarged prostate in the $<40$ years, $40-49$ years, 50 59 years and $\geq 60$ years age groups were $13.46 \%$ (21/156),
$29.17 \%$ (126/432), 53.37\% (190/356) and 78.18\% (448/573), respectively. We can, therefore, speculate that even if serum tPSA levels remain normal, prostatic hyperplasia or hypertrophy may occur. Furthermore, it is important to note that the incidences of prostatic hyperplasia and hypertrophy increase significantly with age.

Our present study also showed that tPSA, \%p2PSA and phi did not increase with age, as reported in most of the existing literature, ${ }^{12,13,18,19}$ and that there was no significant difference in these three biomarkers when compared across men of different ages. This finding differs from previous studies, possibly because of the nature of our study population. In our population of healthy males, we ruled out the possibility of benign prostatic hyperplasia (BPH) and other related diseases. However, the upward trends of tPSA, \%p2PSA and phi described in previous studies concurred with the tendency for BPH to increase with age in our study. we can speculate that the elevated biomarker levels, including those of tPSA and phi, in the healthy population of previous studies was caused by $\mathrm{BPH}$, remains unclear because these studies failed to include the imaging data. Future studies should aim to address this issue.

In the present study, we also observed that tPSA, \% p2PSA and phi showed similar trends with age, peaking at 40-49 years. This can be explained by the histological appearance of BPH after the age of 40 years. Between 
the ages of 40 and 49 , the prostate is more likely to experience enlarged glandular cells (hypertrophy) and hyperplasia. There are no positive features for $\mathrm{BPH}$ in imaging while $\mathrm{BPH}$ is involved in the initial stages of hyperplasia and hypertrophy. Consequently, higher levels of tPSA, \%p2PSA and phi are often observed in this age group. It is also worth mentioning that phi falls to its lowest levels at the age of $\geq 60$ years, which may be associated with a decline in liver and kidney metabolism, estrogen inactivation and androgen secretion, and the attenuation of function in the hypothalamus-pituitary-testicular gonad axis. ${ }^{22}$ Does phi predict a lower risk of prostate disease such as BPH in these older men? Unfortunately, the answer to this question requires further validation. Interestingly, trends in the other three biomarkers (fPSA, \%fPSA and p2PSA) with age were essentially identical, showing peaks $<40$ years of age and then falling gradually with age. p2PSA, one of the truncated forms of ProPSA, is created after cleavage by kallikrein and has been shown to be highly associated with the development of PCa. ${ }^{23}$ In this study, the peak levels of p2PSA were observed in healthy young adults. Furthermore, we established RIs for p2PSA, \%p2PSA and phi in a healthy male population. The RIs of the four age cohorts tested herein were different, and we also identified that the upper and lower limits of reference values for these biomarkers at the age of 40-49 years were generally higher than those at $\geq 60$ years.

There are two primary limitations of this study. First, the study population was recruited from a third-class hospital in Chongqing. Consequently, there may have been a certain level of bias in terms of population selection. Second and most importantly, defining prostate health through laboratory tests and transabdominal prostate ultrasound is clearly insufficient. Clearly, comprehensive prostate health screening should also include direct rectal examination (DRE), transrectal ultrasonography (TRUS), magnetic resonance imaging (MRI) and prostate biopsy.

\section{Conclusion}

This study describes a relation between age and changes in the levels of six markers, including PSA and p2PSA. Interestingly, for the first time, we revealed the significance of determining age-specific p2PSA and phi values in healthy men in Chongqing, China. The typical higher age-specific reference interval for serum p2PSA and phi in comparison with other populations also underlines the need for regional research within individual countries.

\section{Abbreviations}

$\mathrm{BPH}$, benign prostatic hyperplasia; $\mathrm{PCa}$, prostate cancer; PSA, prostate-specific antigen; p2PSA, prostate-specific antigen isoforms 2 .

\section{Ethics approval and informed consent}

This study complied with the Code of Ethics of the World Medical Association and was approved by the Hospital Ethics Committee (Reference Number: 2014-33). All subjects provided signed and informed consent.

\section{Disclosure}

The authors report no conflicts of interest in this work.

\section{References}

1. Siegel RL, Miller KD, Jemal A. Cancer statistics, 2018. CA Cancer J Clin. 2018;68:7-30. doi:10.3322/caac. 21442

2. Attard G, Parker C, Eeles RA, et al. Prostate cancer. Lancet. 2016;387:70e82. doi:10.1016/S0140-6736(14)61947-4

3. Center MM, Jemal A, Lortet-Tieulent J, et al. International variation in prostate cancer incidence and mortality rates. Eur Urol. 2012;61:1079-1092. doi:10.1016/j.eururo.2012.02.054

4. Siegel RL, Miller KD, Jemal A. Cancer statistics, 2015. CA Cancer J Clin. 2015;65(1):5-29. doi:10.3322/caac.21254

5. Tosoian JJ, Ross AE, Sokoll LJ, Partin AW, Pavlovich CP. Urinary biomarkers for prostate cancer. Urol Clin North Am. 2016;43:17-38. doi:10.1016/j.ucl.2015.08.003

6. Huang YQ, Sun T, Zhong WD, Wu CL. Clinical performance of serum [-2]proPSA derivatives, \%p2PSA and phi, in the detection and management of prostate cancer. Am J Clin Exp Urol. 2014;2:343-350.

7. Vukovic I, Djordjevic D, Bojanic N, Babic U, Soldatovic I. Predictive value of [-2]propsa (p2psa) and its derivatives for the prostate cancer detection in the 2.0 to $10.0 \mathrm{ng} / \mathrm{mL}$ PSA range. Int Braz J Urol. 2017;43 (1):48-56. doi:10.1590/S1677-5538.IBJU.2016.0256

8. Al Saidi SS, Al Riyami NB, Al Marhoon MS, et al. Validity of prostate health index and percentage of [-2]pro-prostate-specific antigen as novel biomarkers in the diagnosis of prostate cancer: omani tertiary hospitals experience. Oman Med J. 2017;32(4):275283. doi:10.5001/omj.2017.55

9. Furuya K, Kawahara T, Narahara M, et al. Measurement of serum isoform [-2]proPSA derivatives shows superior accuracy to magnetic resonance imaging in the diagnosis of prostate cancer in patients with a total prostate-specific antigen level of 2-10 ng/ mL. Scand J Urol. 2017;51(4):251-257. doi:10.1080/2168180 5.2017 .1298155

10. Lazzeri M, Briganti A, Scattoni V, et al. Serum index test \%[-2] proPSA and Prostate Health Index are more accurate than prostate specific antigen and \%fPSA in predicting a positive repeat prostate biopsy. J Urol. 2012;188:1137-1143. doi:10.1016/j.juro.2012.06.017

11. Na R, Ye DW, Qi J, et al. Prostate health index significantly reduced unnecessary prostate biopsies in patients with PSA 2$10 \mathrm{ng} / \mathrm{mL}$ and PSA $>10 \mathrm{ng} / \mathrm{mL}$ : results from a Multicenter Study in China. Prostate. 2017;77(11):1221-1229. doi:10.1002/ pros. 23382

12. Sun WG, Liang CZ, Zheng QC, et al. Influence of age on seven putative prostate tumor markers: a cohort study in Chinese men. Asian J Androl. 2017;19:463-467. doi:10.4103/1008-682X.175787 
13. Lacher DA, Hughes JP. Total, free, and complexed prostate-specific antigen levels among US men, 2007-2010. Clin Chim Acta. 2015;448:220-227. doi:10.1016/j.cca.2015.06.009

14. Rhodes T, Jacobson DJ, McGree ME, et al. Distribution and associations of $[-2]$ proenzyme-prostate specific antigen in community dwelling black and white men. J Urol. 2012;187:92-96. doi:10.1016/j. juro.2011.09.017

15. Dixon W. Processing data for ourliers. Biometrics. 1953;9:74-89. doi: $10.2307 / 3001634$

16. Reed AH, Henry RJ, Mason WB. Influence of statistical method used on the resulting estimate of normal range. Clin Chem. 1971;17:275-284.

17. CLSI. Defining, Establishing, and Verifying Reference Intervals in the Clinical Laboratory; Approved Guideline. 3rd ed. CLSI Document C28A3c. Wayne (PA): Clinical and Laboratory Standards Institute; 2008.

18. Liu ZY, Sun YH, Xu CL, et al. Age specific PSA reference ranges in Chinese men without prostate cancer. Asian J Androl. 2008;11:100103. doi:10.1038/aja.2008.17
19. Liu X, Wang J, Zhang S-X, Lin Q. Reference ranges of age-related prostatespecific antigen in men without cancer from Beijing area. Iran J Public Health. 2013;42:1216-1222.

20. Choi H, Park JY, Shim JS, Kim JH, Bae JH. Free prostate-specific antigen provides more precise data on benign prostate volume than total prostate-specific antigen in korean population. Int Nenrourol J. 2013;17(2):73-77. doi:10.5213/inj.2013.17.2.73

21. Pereira J, Bilhim T, Duarte M, et al. Patient selection and counseling before prostatic arterial embolization. Tech Vasc Interv Radiol. 2012;15(4):270-275. doi:10.1053/j.tvir.2012.09.003

22. Inci M, Rifaioglu MM, Inci M, et al. The investigation of total PSA, free PSA, and free/total PSA ratio in patients with liver cirrhosis patients according to Child-Pugh score. Urology. 2013;81(3):617622. doi:10.1016/j.urology.2012.11.022

23. Semjonow A, Köpke T, Eltze E, et al. Pre-analytical in-vitro stability of [-2]proPSA in blood and serum. Clin Biochem . 2010;43(10):926928. doi:10.1016/j.clinbiochem.2010.04.062

\section{Publish your work in this journal}

OncoTargets and Therapy is an international, peer-reviewed, open access journal focusing on the pathological basis of all cancers, potential targets for therapy and treatment protocols employed to improve the management of cancer patients. The journal also focuses on the impact of management programs and new therapeutic agents and protocols on patient perspectives such as quality of life, adherence and satisfaction. The manuscript management system is completely online and includes a very quick and fair peer-review system, which is all easy to use. Visit http://www.dovepress.com/ testimonials.php to read real quotes from published authors. 